\title{
Failure Rate Calculation of PC's SMPSs
}

\author{
B. Abdi, R. Ghasemi, and S. M. M. Mirtalaei, Member, IACSIT
}

\begin{abstract}
Today reliability is one of the serious requirements of electronic systems. The numbers of system failures, repair cost, guarantee, etc are estimated by reliability estimation. In this paper, the reliability of a switching power supply, which is used for personal computers, evaluates. It will show that the most of failure rates depends on power circuit because of more stresses and dissipations. Derating effect of devices on the failure rate will discuss. Part stress count utilizes for failure rate calculations and a prototype's waveforms uses for stress detection and calculation of dissipations.
\end{abstract}

Index Terms - Failure rate, reliability, SMPS, PC.

\section{INTRODUCTION}

Switch Mode Power Supplies (SMPSs) are used in Personal Computers (PCs), because of their high efficiency and low volume and weight. SMPSs are used in all PCs for convert the voltage to different levels and establish it against input voltage and load variations [1].

The efficiency of joinery SMPSs, are used in PCs, is about $70 \%$ and all the power consumption of PC produced by subsystem. So they are the most critical point of PCs from reliability lookout and almost $90 \%$ of PC failures belong to their SMPSs.

Lifetime and failure rate of a system can be predicted by reliability calculations. Recently, reliability became to a prevalent issue in power electronic systems. The effect of transformer leakage inductance on SMPSs reliability discussed in [2], and influence of electrolytic capacitors on SMPS's reliability is given in [3]. Reliability modification of power electronic converter for full cell and photovoltaic application are discussed [4]-[6]. Dissertation of operating mode (continuous and discontinuous modes) with aim of reliability, for SMPSs, presented in [7] and using a single integrated power module (IPM) instead of paralleling power electronic devices are given in [8].

In this paper, the method of reliability calculation is presented for a PC's SMPS. The most critical point of them is introduced and the contrivances for their modifications are proposed.

The reliability calculation are done according to MIL-HDBK-217 [9] and using the method of part stress manner.

\section{DEFINITION OF RELIABILITY}

The probability of proper function of a system after a time

Manuscript received January 23, 2013; revised May 18, 2013.

This work was supported in part by Damavand Branch, Islamic Azad University.

The Authors are with Damavand Branch, Islamic Azad University, Damavand, Tehran, Iran (e-mail: babakabdi@ieee.org). interval is referred to as its reliability.

It is dependent on the type and quality of the parts and materials used in the device, tension of each part endures and the ambient conditions which the devices are working. The failure rate in most of the electronic systems is constant, represented by $\lambda$; the reliability is expressed by:

$$
R(t)=e^{-\lambda t}
$$

The mathematical mean of $R(t)$ occurs at:

$$
t=\frac{1}{\lambda}
$$

Which is the amount of time that should elapses until the first failure occurs. This is called the Mean Time to Failure (MTTF). The mean time to repair (MTTR) of the system is negligible compared to MTTF, so the mean time between failures (MTBF) of a system is expressed as:

$$
M T B F=M T T R+M T T F=\frac{1}{\lambda}
$$

The total rate of the system failure is the sum of the failure rates of all parts of the system:

$$
\lambda_{\text {system }}=\sum_{n=1}^{N} \lambda_{\text {part }}
$$

Hence, the reliability of the system will be the product of all the system components' reliabilities [9]:

$$
R_{\text {system }}=\prod R_{\text {part }}
$$

\section{REliability CALCUlation OF PC's SMPS}

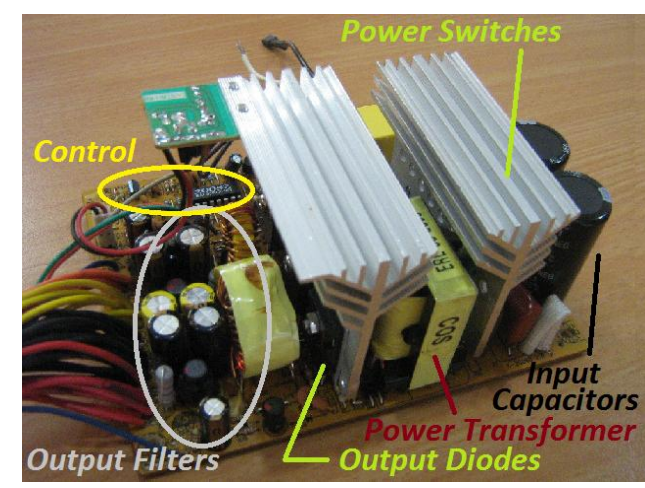

Fig. 1. PC's switch mode power supply.

Power supply, used in PC, convert input voltage (220V, $50 \mathrm{~Hz}$ or $115 \mathrm{~V}, 60 \mathrm{~Hz}$ ) to $\pm 12,+5$ and $+3.3 \mathrm{VDC}$ and regulate them against input voltage and load variations. They can provide up to 200 watts continuously and up to 600 watts instantaneously. A PC's power supply is shown in Fig. 1 and its Block diagram is given in Fig. 2. It is basically a half 
bridge DC-DC converter which stabilizes output voltages using Pulse Width Modulation (PWM) control [1].

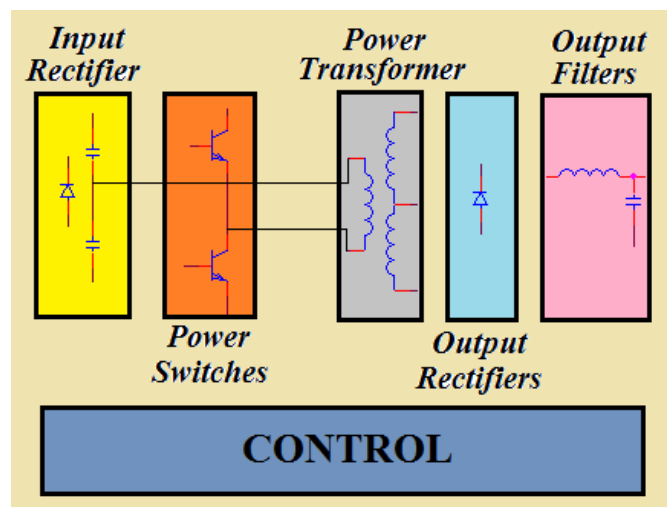

Fig. 2. Block diagram of a switch mode power supply.

In reliability calculation, there is a basic failure rate for each device which is shown by $\lambda_{b}$. The basic failure rate is affected by quality factor of each device $\left(\pi_{Q}\right)$, stress factor $\left(\pi_{S}\right)$ which is the ratio of operational to nominal parameters (like voltage and current), temperature factor $\left(\pi_{T}\right)$ and working ambient or environmental factor $\left(\pi_{E}\right)$. There are another factors belong to specific devices. For example capacitance factor $\left(\pi_{c}\right)$ belongs to capacitors.

All relations, equations and coefficients are achieved from MIL-HDBK-217 [9] and will not repeat in the reliability evaluation below.

According Fig. 1, reliability evaluation can be calculated as follows:

\section{A. Failure Rate of Input Rectifier}

Input rectifier consist a PBU605 bridge $(600 \mathrm{~V}, 6 \mathrm{~A})$ and two electrolytic capacitors $(680 \mathrm{uF}, 200 \mathrm{~V})$ in series. The bridge includes 4 diodes which details of their failure rate calculation are given in section (3-C). Briefly, the failure rate for input bridge rectifier is:

$$
\begin{aligned}
& \lambda_{D i}=\lambda_{b} \pi_{T} \pi_{S} \pi_{Q} \pi_{E} \pi_{C}= \\
& 0.0038 \times 1.4 \times 0.2 \times 8 \times 1 \times 1=0.0085 F / 10^{6} h
\end{aligned}
$$

Half bridge DC-DC converters utilize two serried bulk capacitors in their input (Fig. 2). The failure rate of electrolytic capacitors calculates as:

$$
\lambda_{c}=\lambda_{b} \pi_{T} \pi_{C} \pi_{V} \pi_{S R} \pi_{Q} \pi_{E}
$$

$\pi_{C}$ is capacitance factor depends to amount of capacitance, $\mathrm{C}$, by equation (7).

$$
\pi_{C}=C^{0.23}
$$

For a $680 \mathrm{uF}$ capacitor, $\pi_{C}$ is 4.5 .

$\pi_{V}$ is voltage stress factor. It depends on the applied voltage to nominal voltage ratio, $\mathrm{S}$, by equation (8).

$$
\pi_{V}=\left(\frac{S}{0.6}\right)^{17}+1
$$

Half of input rectified voltage, $160 \mathrm{~V}$, will be applied to each capacitor. So, $S=0.8(160 / 200)$ and $\pi_{V}=134$.

$\pi_{S R}$ is series resistance factor. So, the failure rate of two input electrolytic capacitors is:

$$
\begin{aligned}
& \lambda_{c}=\lambda_{b} \pi_{T} \pi_{C} \pi_{V} \pi_{S R} \pi_{Q} \pi_{E}= \\
& 0.0004 \times 1.6 \times 4.5 \times 134 \times 3.3 \times 3 \times 1=0.85 F / 10^{6} h
\end{aligned}
$$

\section{B. Failure of Power Switches and Transformer}

Half bridge converter consist two power switches. Here two power BJTs, E13009KA, are used for this aim. The failure rate of BJTs can be calculated by equation (9).

$$
\lambda_{t}=\lambda_{b} \pi_{T} \pi_{A} \pi_{R} \pi_{S} \pi_{Q} \pi_{E}
$$

$\pi_{A}$ is application factor, 0.7 for switching applications. $\pi_{R}$ is power rating factor. $\pi_{S}$ is voltage stress factor and depends on applied voltage to rated voltage ratio (Vs) by:

$$
\pi_{s}=0.045 e^{\left(3.1 V_{s}\right)}
$$

$\pi_{T}$ is temperature factor determines by:

$$
\pi_{T}=\exp \left[-2114 \times\left(\frac{1}{T_{J}+273}-\frac{1}{298}\right)\right]
$$

where $T_{J}$ is junction temperature of transistor and calculates as follow:

$$
T_{J}=T_{C}+\theta_{J C} \times P_{\text {loss }}
$$

where $T_{C}$ is transistor case temperature, $\theta_{J C}$ is thermal resistance between junction and case and $P_{\text {loss }}$ is transistor dissipation. In switching application transistor has two kind of dissipation: static $\left(V_{C E} . I_{C}\right)$ and dynamic.

Dynamic loss originated by voltage and current overlap and calculated by integral of their multiplication during switching transient. Transformer's leakage inductance cause ignorable overlap between voltage and current during on transient as well as high dissipation during off transient.

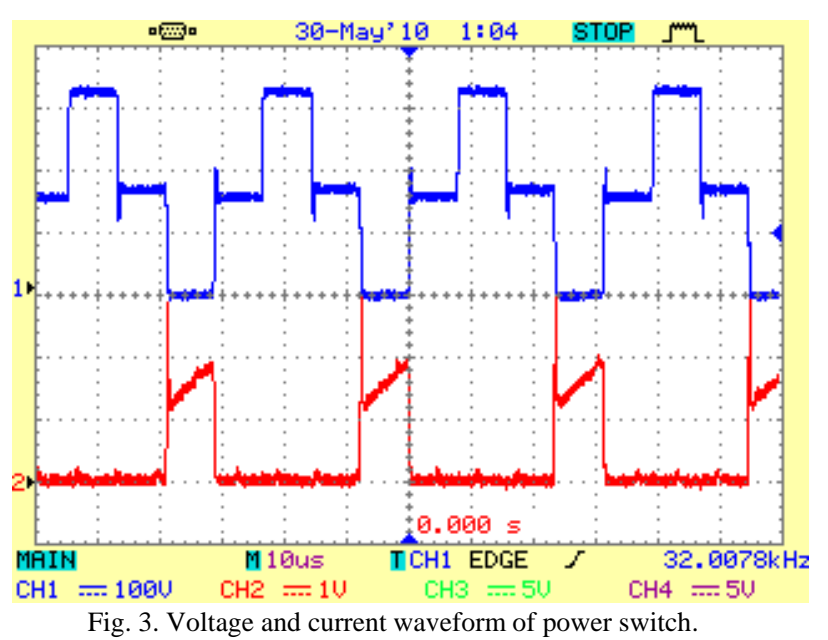

Fig. 3 illustrates the voltage and current wave forms of switches for a sample commercial power supply, and their 
overlap during off transient is shown in Fig. 4.

Transistors' saturation voltage $\left(V_{C E(s a t)}\right)$ is 1.5 volts. So, According to current shape and duty cycle given in Fig. 3, the static loss is $0.56 \mathrm{~W}\left(V_{C E(s a t)} . I_{C(A v g)}\right.$. D). according Fig. 4, dynamic loss is $1.76 \mathrm{~W}$. Therefore the total loss is $2.32 \mathrm{~W}$ $(0.56+1.76)$. Transistor case temperature is $60^{\circ} \mathrm{C}$ and according to transistor's datasheet, thermal resistance between junction and case is $0.6{ }^{\circ} \mathrm{C} / \mathrm{W}$.

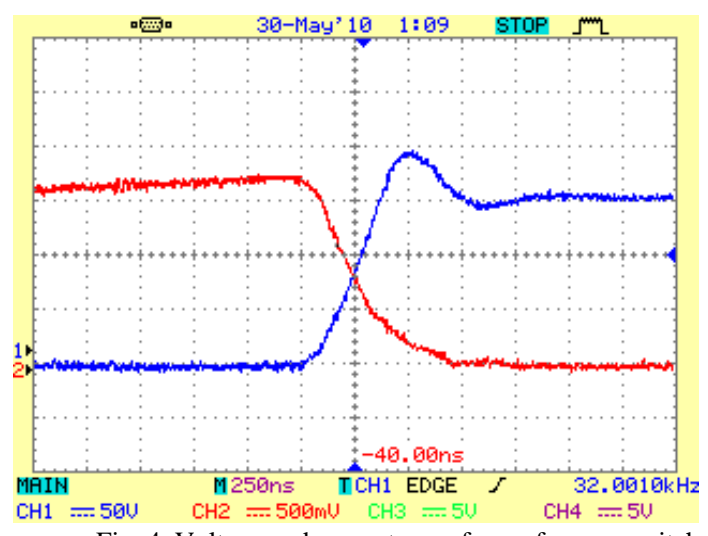

Fig. 4. Voltage and current waveform of power switch.

Substituting above numbers in equations 12 and 11 results $\pi_{T}=2.2$.

Nominal voltage of transistors is $400 \mathrm{~V}$ and according Fig. 3 , applied voltage is $320 \mathrm{~V}$. These results $V s=0.8$ and $\pi_{S}=0.54$.

Finally, the failure rate of power switches can be determined as:

$$
\begin{aligned}
& \lambda_{t}=\lambda_{b} \pi_{T} \pi_{A} \pi_{R} \pi_{S} \pi_{Q} \pi_{E}= \\
& 0.00074 \times 2.2 \times 0.7 \times 5.5 \times 0.54 \times 8 \times 1=0.027 \mathrm{~F} / 10^{6} \mathrm{~h}
\end{aligned}
$$

Transformer's failure rate calculates as follow:

$$
\lambda_{T}=\lambda_{b} \pi_{Q} \pi_{E}=0.0035 \times 30 \times 1=0.1 F / 10^{6} h
$$

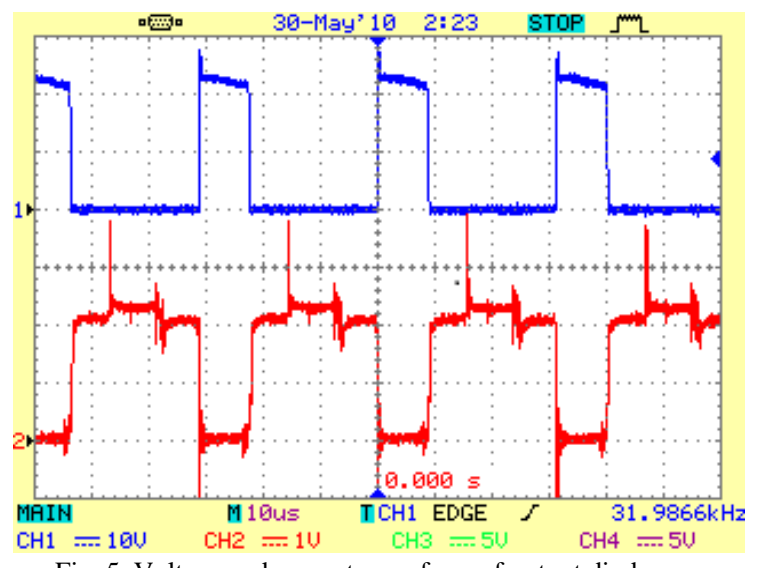

Fig. 5. Voltage and current waveform of output diodes.

\section{Failure Rate of Output Rectifiers}

Double schottky diodes, SB3030PT (30A-30V), are used for high frequency output rectifiers. The failure rate of each diode calculates as:

$$
\lambda_{D 5}=\lambda_{b} \pi_{T} \pi_{S} \pi_{C} \pi_{Q} \pi_{E}
$$

$\pi_{C}$ is contact construction factor. It is 1 for metallurgically bonded construction. Fig. 5 illustrates voltage and current waveforms for $+5 \mathrm{~V}$ output. According to this figure, applied voltage is 14 volts which results $\pi_{S}=0.11$. So, the failure rate of each diode in $+5 \mathrm{~V}$ rectifier is:

$$
\begin{aligned}
& \lambda_{D 5}=\lambda_{b} \pi_{T} \pi_{S} \pi_{C} \pi_{Q} \pi_{E}= \\
& 0.003 \times 3 \times 0.11 \times 1 \times 8 \times 1=0.008 F / 10^{6} \mathrm{~h}
\end{aligned}
$$

Applied voltage for $+12 \mathrm{~V}$ and $-12 \mathrm{~V}$ is $27 \mathrm{~V}$ which results $\pi_{S}=0.7$ and $\lambda_{D 12}=0.05 \mathrm{~F} / 10^{6} \mathrm{~h}$.

\section{Failure Rate of Output Filters}

Each output consist a filer including two capacitors and two inductors. The failure rate of electrolytic capacitor calculates by equation 6 . Two $10 \mathrm{~V}, 2200 \mathrm{UF}$ capacitor are used in $+5 \mathrm{~V}$ output. So, failure rate of capacitors for this output calculates as:

$$
\begin{aligned}
& \lambda_{c 5}=\lambda_{b} \pi_{T} \pi_{C} \pi_{V} \pi_{S R} \pi_{Q} \pi_{E}= \\
& 0.0004 \times 1.6 \times 5.9 \times 1 \times 3.3 \times 3 \times 1=0.037 F / 10^{6} h
\end{aligned}
$$

Two $16 \mathrm{~V}, 1000 \mathrm{UF}$ capacitor are used in $+12 \mathrm{~V}$ output. So, failure rate of capacitors for this output calculates as:

$$
\begin{aligned}
& \lambda_{c 12}=\lambda_{b} \pi_{T} \pi_{C} \pi_{V} \pi_{S R} \pi_{Q} \pi_{E}= \\
& 0.0004 \times 1.6 \times 4.9 \times 45.5 \times 3.3 \times 3 \times 1=1.41 F / 10^{6} h
\end{aligned}
$$

Two 16V, 470UF capacitor are used in $-12 \mathrm{~V}$ output. So, failure rate of capacitors for this output calculates as:

$$
\begin{aligned}
& \lambda_{c m 12}=\lambda_{b} \pi_{T} \pi_{C} \pi_{V} \pi_{S R} \pi_{Q} \pi_{E}= \\
& 0.0004 \times 1.6 \times 4.1 \times 45.5 \times 3.3 \times 3 \times 1=1.18 F / 10^{6} h
\end{aligned}
$$

Failure rate of inductance calculates as:

$$
\lambda_{t}=\lambda_{b} \pi_{c} \pi_{Q} \pi_{E}
$$

It is the same for entire outputs and calculates as:

$$
\begin{aligned}
& \lambda_{t}=\lambda_{b} \pi_{c} \pi_{Q} \pi_{E}= \\
& 0.0006 \times 1 \times 20 \times 1=0.012 \mathrm{~F} / 10^{6} \mathrm{f}
\end{aligned}
$$

\section{E. Failure Rate of Control Circuit}

Control unit includes a control IC, 60 resistors, 15 ceramic capacitors and 8 electrolytic types, 10 diodes (1n4148) and 5 transistors (2N2222).

\section{1) Failure Rate of Control IC}

It calculates as:

$$
\lambda_{I C}=\left(C_{1} \pi_{T}+C_{2} \pi_{E}\right) \pi_{Q} \pi_{L}
$$

where $C 1$ is a coefficient based on the number of transistor used in the $I C$. $C 2$ is packaging failure rate and $\pi_{L}$ is leaming factor and depends on producer background. These entire coefficients are given in [9]. Therefore IC's failure rate can be calculated as:

$$
\lambda_{I C}=(0.01 \times 0.71+0.0079 \times 2) 10 \times 1=0.229
$$

Failures per 106 hours (F/106h) 


\section{2) Failure Rate of Control's Resistors}

There are 60 resistors in the control circuit. Although they have different values, they are the same in reliability calculation.

The Failure rate of each resistor can be calculated as:

\section{3) Failure rate of control's capacitors}

Ceramic capacitor's failure rate calculates as:

$$
\lambda_{c}=\lambda_{b} \pi_{C V} \pi_{Q} \pi_{E}
$$

$\pi_{C V}$ is capacitance factor. Failure rate for each ceramic capacitor is:

$$
\lambda_{c}=0.00075 \times 1 \times 10 \times 2=0.015\left(F / 10^{6} h\right)
$$

15 ceramic capacitors are used in control circuit. So their entire failure rate is:

$$
\lambda_{c}=15 \times 0.015=0.225\left(F / 10^{6} h\right)
$$

There are also 8 electrolytic capacitors $(10 \mathrm{uF}, 50 \mathrm{~V})$ in control circuit. The failure rate for this kind of capacitors calculates as:

$$
\begin{gathered}
\lambda_{c}=\lambda_{b} \pi_{T} \pi_{C} \pi_{V} \pi_{S R} \pi_{Q} \pi_{E} \\
\lambda_{C}=0.0004 \times 1.6 \times 1.9 \times 1 \times 3.3 \times 3 \times 1=0.012 F / 10^{6} h
\end{gathered}
$$

\section{4) Failure rate of control's diodes}

There are 10, 1N4148, diodes in control circuit. Detail of calculation process is given in section (14). Briefly, the failure rate for these diodes is:

$$
\begin{aligned}
& \lambda_{D C}=\lambda_{b} \pi_{T} \pi_{S} \pi_{Q} \pi_{E} \pi_{C}= \\
& 0.0038 \times 1.4 \times 0.054 \times 8 \times 6 \times 2=0.0257 F / 10^{6} h
\end{aligned}
$$

\section{5) Failure rate of control's transistors}

There are 5, 2N2222, transistors in control circuit. Detail of calculation process is given in section (9). Briefly, the failure rate for these entire transistors is:

$$
\begin{aligned}
& \lambda_{T C}=\lambda_{b} \pi_{T} \pi_{A} \pi_{R} \pi_{S} \pi_{Q} \pi_{E}= \\
& 0.00074 \times 1.6 \times 1.5 \times 0.43 \times 0.11 \times 6=0.0004 F / 10^{6} h
\end{aligned}
$$

\section{DISCUSSION}

Failure rate of different parts of a case study switch mode power supply are given in Table I. It is clear that the power circuitry have much failure rate than control circuit. Although the number of parts in control is more than power

\begin{tabular}{|c|c|c|c|c|}
\hline & & 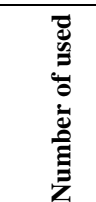 & $\begin{array}{l}\text { Each part failure rate } \\
\lambda_{\text {each }}\end{array}$ & $\mathbf{z}$ \\
\hline \multirow{11}{*}{ 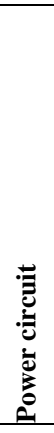 } & Input Rectifier & 4 & 0.008562 & 0.034 \\
\hline & Input Cap. & 2 & 0.85 & 0.17 \\
\hline & Power Switch & 2 & 0.027 & 0.054 \\
\hline & +5V Diode & 2 & 0.008 & 0.016 \\
\hline & 12V Diode & 4 & 0.05 & 0.2 \\
\hline & +5V Cap. & 2 & 0.037 & 0.074 \\
\hline & +12V Cap. & 2 & 1.41 & 2.82 \\
\hline & -12V Cap. & 2 & 1.18 & 2.36 \\
\hline & Inductors & 5 & 0.012 & 0.06 \\
\hline & Power Trans. & 1 & 0.027 & 0.027 \\
\hline & $\begin{array}{l}\text { Power stage } \\
\text { circuit }\end{array}$ & \multicolumn{3}{|c|}{$\lambda_{\text {power }}=5.8$} \\
\hline \multirow{7}{*}{ 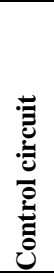 } & IC & 1 & 0.229 & 0.229 \\
\hline & resistors & 60 & 0.0035 & 0.21 \\
\hline & Ceramic Cap. & 15 & 0.015 & 0.225 \\
\hline & Electrolytic Cap. & 8 & 0.012 & 0.096 \\
\hline & Transistors & 5 & 0.0004 & 0.002 \\
\hline & Diodes & 10 & 0.026 & 0.26 \\
\hline & Control circuit & \multicolumn{3}{|c|}{$\lambda_{\text {control }}=1.022$} \\
\hline \multirow{2}{*}{\multicolumn{2}{|c|}{ Total system }} & \multicolumn{3}{|c|}{$\lambda_{\text {system }}=\sum_{n=1}^{N} \lambda_{\text {part }}=6.82$} \\
\hline & & \multicolumn{3}{|c|}{$M T B F=\frac{1}{\lambda}=146627(h / f)$} \\
\hline
\end{tabular}
circuit, the failure rate of power circuit is more because of more stress and dissipation.

In the power circuit $12 \mathrm{~V}$ electrolytic capacitors have more failure rate because of voltage derating disobedience.

In control circuit, electrolytic capacitors have a little failure rate because of good voltage derating. Their nominal voltage is $50 \mathrm{~V}$ and their applied voltage $12 \mathrm{~V}$. So, reliability can be modified by proper derate of capacitors.

\section{CONCLUSION}

The reliability of a PC's SMPS has been fully derived in this paper. It is shown that the elements of the power circuit, account for the largest portion of the failure rate in the converter, and to increase the reliability, the power circuit elements should be focused on. Calculations for a prototype power supply have shown that electrolytic capacitors are more vulnerable because of weak voltage derating.

\section{REFERENCES}

[1] A. Pressman, "Switching Power Supply Design,"2nd ed. McGraw-Hill, 1998.

[2] B. Abdi, M. B. Menhaj, L. Yazdanparast, and J. Milimonfared, "The effect of the transformer winding on the reliability of switching power supplies," IEEE, 2006.

[3] Y. M. Chen, H. C. Wu, M. W. Chou, and K. Y. Lee, "Online failure prediction of the electrolytic capacitor for LC filter of switching-mode power converters," IEEE Transactions on Industrial Electronics, vol. 55, no. 1, January 2008.

[4] C. Rodriguez and G. A. J. Amaratunga, "Long-lifetime power inverter for photovoltaic AC modules," IEEE Transactions on Industrial Electronics, vol. 55, no. 7, July 2008.

[5] F. Chan and H. Calleja, "Design strategy to optimize the reliability of grid-connected PV systems", IEEE Transactions on Industrial Electronics, vol. 56, no. 11, November 2009.

[6] A. H. Ranjbar, B. Abdi, S. A. Nabavi Niak, G. B. Gharehpetian, and J Milimonfared, "Reliability comparison of fuel-cell DC-DC converter in two cases of using IPM switch and paralleling MOSFETs," International Symposium on Power Electronics, Electrical Drives, Automation and Motion, SPEEDAM, 2008.

[7] B. Abdi, A. H. Ranjbar, J Milimonfared, and G. B. Gharehpetian, "Reliability comparison of boost PFC converter in DCM and CCM operating modes," International Symposium on Power Electronics, Electrical Drives, Automation and Motion, SPEEDAM, 2008.

[8] B. Abdi, A. H. Ranjbar, G. B. Gharehpetian, and J. Milimonfared, "Reliability considerations for parallel performance of semiconductor switches in high power switching power supplies," IEEE Transactions on Industrial Electronics, vol. 56, no. 6, June 2009. 
[9] Reliability prediction of electronic equipment, Military Handbook, MIL-HDBK-217, Dec. 2, 1991.

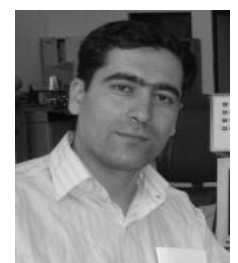

Babak Abdi was born in Tehran in 1976. He received his M.S. and Ph.D. degree in electrical engineering in 2005 and 2009 from Amirkabir University of Technology (Tehran Polytechnic), Tehran, Iran, respectively. He is currently a member of IEEE and a faculty member of Damavand branch, Islamic Azad University, Tehran, Iran. His research interests include power electronics, application of reliability in power electronics, Electromagnetic Interferences (EMI), and electrical machines and drives.

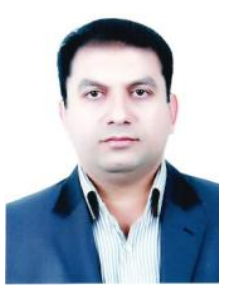

Reza Ghasemi was born in Tehran, Iran in 1979. He received his B.Sc. degree in Electrical engineering from Semnan University in 2000. He received the M.Sc. and the Ph.D. degree in control engineering from Amirkabir University of Technology, Tehran, Iran in 2004 and 2009, respectively. His research interests include large-Scale Systems, adaptive control, robust control, nonlinear control, and intelligent systems. Reza Ghasemi joined the Department of Electrical Engineering, Damavand Branch, Islamic Azad University, Tehran, Iran, where he is currently an assistant professor of electrical engineering.

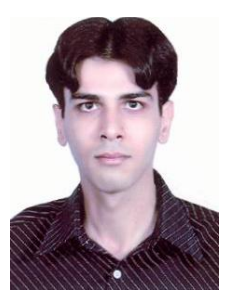

Sayyed Mohammad Mehdi Mirtalaei was born in Shahreza-Isfahan, Iran in 1983. He received his B.S. degree in electrical engineering from Isfan University of Technology, Iran in 2005. He received his M.S. and $\mathrm{Ph} . \mathrm{D}$. in electrical engineering from Amirkabir University of Technology, Tehran, Iran in 2007 and 2012 respectively. His research interest are power electronics, EMI/EMC and numerical method in electromagnetic. 